\title{
Longitudinal genomic surveillance of multidrug-resistant Escherichia coli carriage in a long-term care facility in the United Kingdom
}

Hayley J. Brodrick ${ }^{1 *+}$, Kathy E. Raven ${ }^{1+}$, Teemu Kallonen², Dorota Jamrozy ${ }^{2}$, Beth Blane ${ }^{1}$, Nicholas M. Brown ${ }^{3,4,6}$, Veronique Martin ${ }^{4,5}$, M. Estée Török ${ }^{1,3,6}$, Julian Parkhill ${ }^{2}$ and Sharon J. Peacock ${ }^{1,2,6,7}$

\begin{abstract}
Background: Residents of long-term care facilities (LTCF) may have high carriage rates of multidrug-resistant pathogens, but are not currently included in surveillance programmes for antimicrobial resistance or healthcareassociated infections. Here, we describe the value derived from a longitudinal epidemiological and genomic surveillance study of drug-resistant Escherichia coli in a LTCF in the United Kingdom (UK).
\end{abstract}

Methods: Forty-five of 90 (50\%) residents were recruited and followed for six months in 2014. Participants were screened weekly for carriage of extended-spectrum beta-lactamase (ESBL) producing E. coli. Participants positive for ESBL E. coli were also screened for ESBL-negative E. coli. Phenotypic antibiotic susceptibility of E. coli was determined using the Vitek2 instrument and isolates were sequenced on an Illumina HiSeq2000 instrument. Information was collected on episodes of clinical infection and antibiotic consumption.

Results: Seventeen of 45 participants (38\%) carried ESBL E. coli. Twenty-three of the 45 participants (51\%) had 63 documented episodes of clinical infection treated with antibiotics. Treatment with antibiotics was associated with higher risk of carrying ESBL E. coli. ESBL E. coli was mainly sequence type (ST)131 (16/17, 94\%). Non-ESBL E. coli from these 17 cases was more genetically diverse, but ST131 was found in eight (47\%) cases. Whole-genome analysis of 297 ST131 E. coli from the 17 cases demonstrated highly related strains from six participants, indicating acquisition from a common source or person-to-person transmission. Five participants carried highly related strains of both ESBL-positive and ESBL-negative ST131. Genome-based comparison of ST131 isolates from the LTCF study participants with ST131 associated with bloodstream infection at a nearby acute hospital and in hospitals across England revealed sharing of highly related lineages between the LTCF and a local hospital.

Conclusions: This study demonstrates the power of genomic surveillance to detect multidrug-resistant pathogens and confirm their connectivity within a healthcare network.

Keywords: ESBL, ST131, Genome, Sequence, Phylogeny

\footnotetext{
* Correspondence: hjb60@medschl.cam.ac.uk

${ }^{\dagger}$ Equal contributors

'Department of Medicine, University of Cambridge, Box 157, Addenbrooke's

Hospital, Hills Road, Cambridge CB2 0QQ, UK

Full list of author information is available at the end of the article
} 


\section{Background}

Escherichia coli is a leading cause of bloodstream and urinary tract infections $[1,2]$. In the United Kingdom (UK), there has been an increase in E. coli bloodstream infections since 2008, with a $16.7 \%$ rise in cases between 2010 and 2014 [3]. The uropathogenic E. coli (UPEC) lineage sequence type (ST)131 was initially reported in several countries during 2008 [4-6] and has since become widely disseminated. E. coli ST131 frequently carries plasmid-mediated extended-spectrum betalactamase (ESBL) genes that confer resistance to thirdgeneration cephalosporins [7-9]. Infection with ESBL $E$. coli is associated with increased hospital stay, healthcare costs and mortality compared with infections caused by non-ESBL E. coli [10-12]. The most frequently identified ESBL gene in E. coli ST131, bla $a_{\mathrm{CTX}-\mathrm{M}-15}$, is globally disseminated and predominates in this ST in North America $[6,7]$, the UK [13], Europe [14-16], Asia [17, 18] and South America [19]. E. coli bloodstream isolates submitted between 2001 and 2010 to the British Society for Antimicrobial Chemotherapy (BSAC) Bacteraemia Resistance Surveillance Programme demonstrate the prevalence of this lineage within the UK [20]. Clonal complex (CC) 131 accounted for $12 \%$ of isolates and contained $81.7 \%$ of all blaCTX-M-1 group (which includes bla $a_{\mathrm{CTX}}$ M-15) genes [20].

Global surveillance of antibiotic consumption and the emergence of resistance is gathering pace through numerous initiatives, including those by the World Health Organization (WHO), European Centre for Disease Prevention and Control (ECDC) and the Department of Health [21-25]. Target species include E. coli, particularly $E$. coli that are resistant to third-generation cephalosporins and fluoroquinolones. The development of surveillance frameworks requires consideration of the target populations.

The global increase in life expectancy has been associated with a rise in the number of people requiring care in long-term care facilities (LTCFs). LTCFs also provide increasing levels of post-acute, rehabilitative and palliative care to optimise patient flow through acute hospitals $[26,27]$. Point prevalence studies have reported carriage rates of multidrug-resistant $E$. coli in residents of LTCF in excess of $50 \%$ in Ireland and $40 \%$ in the UK $[28,29]$. This is significantly higher than the general population, with a recent meta-analysis of community carriage rates reporting a pooled prevalence of ESBL carriage of $2 \%$ in the Americas, $4 \%$ in Europe, $15 \%$ in the eastern Mediterranean and 22\% in South East Asia and Africa [30].

Although high rates of ESBL E. coli in LTCFs have been established, genomic characterisation of the associated isolates has been limited and published studies have utilised molecular techniques such as pulsed-field gel electrophoresis and polymerase chain reaction (PCR)- based assays [28, 29, 31-33], which lack the discrimination of whole-genome sequencing (WGS). Here, we describe a longitudinal study of ESBL-E. coli carriage by residents of a LTCF, in which we determine the frequency of E. coli ST131 isolates and of non-ESBL E. coli isolates in the same patients. Analysis of WGS data for 399 E. coli isolates provided a detailed genetic understanding of the relationships between ESBL-positive and ESBL-negative E. coli within and between study participants. We extended this analysis by comparing 297 ST131 study genomes with more than 200 ST131 genomes of bacteria associated with bloodstream infection in patients across England to place our LTCF isolates into a broader genetic context.

\section{Methods}

Study design, setting and participants

A prospective observational cohort study was conducted during a six-month period in 2014 at a LTCF in Cambridgeshire in the UK, details of which have been published previously in relation to the study of Enterococcus faecium [34]. In brief, the LTCF had 105 beds and was sub-divided into five separate units to which residents were assigned based on cognitive impairment and physical disability.

\section{Sampling, microbiology and data collection}

All residents admitted to the LTCF during the study period were eligible for inclusion. Residents were excluded if they refused consent, were on an end-of-life care pathway or were strongly resistant to basic personal care. Healthcare staff collected stool and urine specimens weekly from study participants, which were processed within $24 \mathrm{~h}$ ( $48 \mathrm{~h}$ at weekends). A total of $10 \mu \mathrm{l}$ of each sample was plated either directly (urine samples only) or following overnight enrichment in $5 \mathrm{~mL}$ of Tryptic Soy broth (Sigma-Aldrich, St Louis, MO, USA) supplemented with cefpodoxime (Oxoid, Basingstoke, $\mathrm{UK}$ ) at $1 \mu \mathrm{g} / \mathrm{mL}$ onto Brilliance ESBL agar (Oxoid, Basingstoke, UK) and incubated at $37{ }^{\circ} \mathrm{C}$ in air for $24 \mathrm{~h}$. Putative ESBL E. coli colonies based on colony colour on chromogenic agar were speciated using matrix-assisted laser desorption/ionization time-of-flight mass spectrometry (MALDI-TOF) (Bruker Daltoniks, Bremen, Germany). A single colony from each positive sample was taken forward for further testing. Antimicrobial susceptibility was determined using the Vitek2 instrument (BioMérieux, Marcy l'Etoile, France) with the N206 card. Expression of ESBL was confirmed using the ESBL and AmpC Detection Disc Set (D68C1, Mast Group, Bootle, UK). All stools positive for ESBL-E. coli were cultured for non-ESBL E. coli by plating $10 \mu \mathrm{L}$ of stool onto Brilliance UTI agar (Oxoid, Basingstoke, UK) and incubating at $37{ }^{\circ} \mathrm{C}$ in air for $24 \mathrm{~h}$. Presumptive E. coli colonies 
were sub-cultured onto Columbia Blood Agar with the addition of a $10 \mu \mathrm{g}$ cefpodoxime disc (Oxoid, Basingstoke, UK). Colonies growing at the edge of the zone of inhibition were selected for identification and antimicrobial susceptibility testing as above. Data were collected from participant nursing care plans and medical records on episodes of infection and antimicrobial consumption. Statistical analysis was performed using STATA v13.1 (STATA, College Station, TX, USA).

\section{Bacterial sequencing and analysis}

Genomic DNA was extracted from single colonies using the QIAxtractor (QIAgen, Hilden, Germany). Library preparation was conducted according to the Illumina protocol, and sequencing was performed on an Illumina HiSeq2000 with 100-cycle paired-end runs. Ninety-six samples were multiplexed per lane to give an average depth of coverage of $\sim 90$-fold. Sequence data have been submitted to the European Nucleotide Archive (ENA) under the accession numbers listed in Additional file 1.

Sequence reads were assembled using Velvet v1.2 [35] and VelvetOptimser v2.2.5 (http://www.vicbioinformatics.com/software.velvetoptimiser.shtml). Assembly improvement was performed using the assembly with the best N50 and SSPACE was used for contig scaffolding [36]. GapFiller was used to close sequence gaps [37] and annotation was performed using PROKKA v1.11 [38] and a genus specific database from RefSeq [39]. STs were identified from the sequence data using the Warwick MLST database [40] and an in-house script [41, 42]. Sequence reads for ST131 isolates were mapped to the $E$. coli reference genome NCTC13441 (European Nucleotide Archive [ENA] accession number ERS530440) using SMALT v0.7.4 [43]. Variants were detected using samtools mpileup v0.1.19 [44] and the parameters '- $\mathrm{d} 1000$ DsugBf' and bcftools v0.1.19, giving a BCF file of all variant sites. A variant quality score of greater than 50 and mapping quality of greater than 30 was used. The majority base call was required to be present in more than $75 \%$ of reads with a minimum mapping of four reads, with at least two mapping to each strand. A pseudogenome was created by substituting bases called at each site in the BCF file into the reference genome. Any sites deemed uncertain following quality scoring were substituted with an $\mathrm{N}$, along with any deletions identified in the context of the reference genome. To create a 'core' genome, mobile genetic regions were masked if they were annotated as predicted phage-, plasmid-, insertion sequence (IS)- or transposon-related genes, or if PHAST identified a putative prophage [45]. Gubbins was used to identify and remove recombination within each genome, giving the final 'core' genome [46], and maximum likelihood phylogenies were created using RAxML with 100 bootstraps and a mid-point root [47].
Trees were visualised using FigTree (v1.4.2) [48] and iTOL (v3) [48, 49].

The presence of antimicrobial resistance genes was determined by comparison of genomes to an in-house database using ResFinder [50]. fimH alleles were identified using in silico PCR and primers detailed in Weissman et al. [51] and Colpan et al. [52]. Seaview was used to curate and assign fimH types, detect fluroquinolone resistance mutations and SNPs associated with $\mathrm{CO}$ and C2 [53]. Analysis of the accessory (non-core) genome composition was conducted as described previously [54]. Distribution of a mobile genetic element (MGE) among the isolates was determined through assembly alignment with MUMmer [55] and with sequence read mapping using SRST 2 [56].

Two further whole-genome sequence datasets were retrieved from the European Nucleotide Archive: (1) 75 ST131 isolates associated with bloodstream infection in patients at the Cambridge University Hospitals NHS Foundation Trust between 2006-2012 (Project PRJEB4681); and (2) ST131 isolates associated with bloodstream infection in ten hospitals in England between 2001 and 2011, submitted to the British Society for Antimicrobial Chemotherapy Resistance Surveillance Project $(n=146$, Project PRJEB4681).

\section{Results \\ Study participants}

Forty-five of 90 (50\%) eligible residents were recruited to the study. The median age of study participants was 82 years (range $=40-104$ years, interquartile range $[\mathrm{IQR}]=71-87$ years), and $29(64 \%)$ were women. Three participants were lost to follow-up because of death $(\mathrm{n}=$ 2 ) or transfer elsewhere $(n=1)$. The median duration of residence in the LTCF by the time the study began was 16 months (range $=5$ days -54 months, IQR $=6-41$ months). Twenty-nine percent $(n=13)$ of recruited residents had the capacity to consent for themselves to take part. The remaining $71 \%(\mathrm{n}=32)$ were recruited following discussion with a resident's consultee who considered whether the resident in question would agree themselves to take part if they had the capacity to do so. Stool samples were collected at recruitment and then at least one week apart until the end of the study period, discharge from the LTCF or death.

\section{Infective episodes and antibiotic consumption}

Debilitated patients are more prone to bacterial infection and antibiotic consumption selects for antibiotic resistant bacteria. In light of this, we collected information on episodes of infection and antibiotic consumption during the study and antibiotic consumption in the 12-month period preceding this. During the study, 23/45 (51\%) participants had 63 documented infective episodes 
$($ median $=1$, range $=1-5, \mathrm{IQR}=1-3.5)$. Infections of the urinary tract were the most common $(\mathrm{n}=33,54 \%)$, followed by those affecting the respiratory tract $(n=16)$ and skin and soft tissue $(n=8)$. The focus of infection was not specified in four cases. Diagnoses were based on clinical features alone with the exception of urinary tract infections, which were investigated using urinalysis to detect leucocytes and nitrites. No clinical specimens were collected from the study cohort for microbiological culture. All episodes were treated with at least one course of empiric antibiotics (median $=2$ courses per patient, range $=1-6, I Q R=1-4)$. The most frequent antibiotics used were trimethoprim (16/63 infective episodes, $25 \%)$, co-amoxiclav $(14 / 63,22 \%)$ and flucloxacillin (10/ $63,16 \%)$. In addition, two catheterised residents (participants $\mathrm{P} 4$ and P6) were on long-term prophylactic antibiotics (trimethoprim and metronidazole, respectively). Antibiotic consumption was also common in the 12 months prior to enrolment, with $31 / 45$ (68.9\%) participants receiving a total of 83 courses of antibiotics (median $=3$, range $=1-5, \mathrm{IQR}=1-4)$. The three most frequently prescribed antibiotics prior to enrolment were trimethoprim $(23 / 83,28 \%)$, co-amoxiclav $(21 / 83,25 \%)$ and amoxicillin (11/83, 13\%).

\section{E. coli carriage}

ESBL E. coli was cultured from stool from 17/45 participants (38\%) (isolated from 241/691 stool samples tested), none of whom were known previously to be ESBL E. coli carriers. Figure 1 shows the timeline for positive and negative samples. Most ESBL-positive E. coli participants were positive on the first stool tested and were carriers thereafter. The exceptions were $\mathrm{P} 9$, who became positive after 13 negative samples, and P16, who became negative after 13 ESBL-positive E. coli samples. Urine was also cultured if a urinary catheter was present. Two of the three catheterised participants (P3 and P6) had ESBL E. coli isolated from urine (3/4 samples and 18/18 samples, respectively), both of whom carried ESBL E. coli in stool. The third case (P4) had ESBL-negative E. coli in both urine and stool.

Almost one-half $(7 / 17,41 \%)$ of ESBL-positive E. coli participants lived in unit 3, which provided residential and minor nursing care for residents with dementia. The majority $(15 / 17,88 \%)$ of ESBL-positive E. coli participants had received at least one course of antibiotics in the 12 months prior to study enrolment, compared with 16/28 (57\%) participants who were ESBL-negative. Antibiotic consumption on at least one occasion was associated with higher risk of having ESBL E. coli isolated

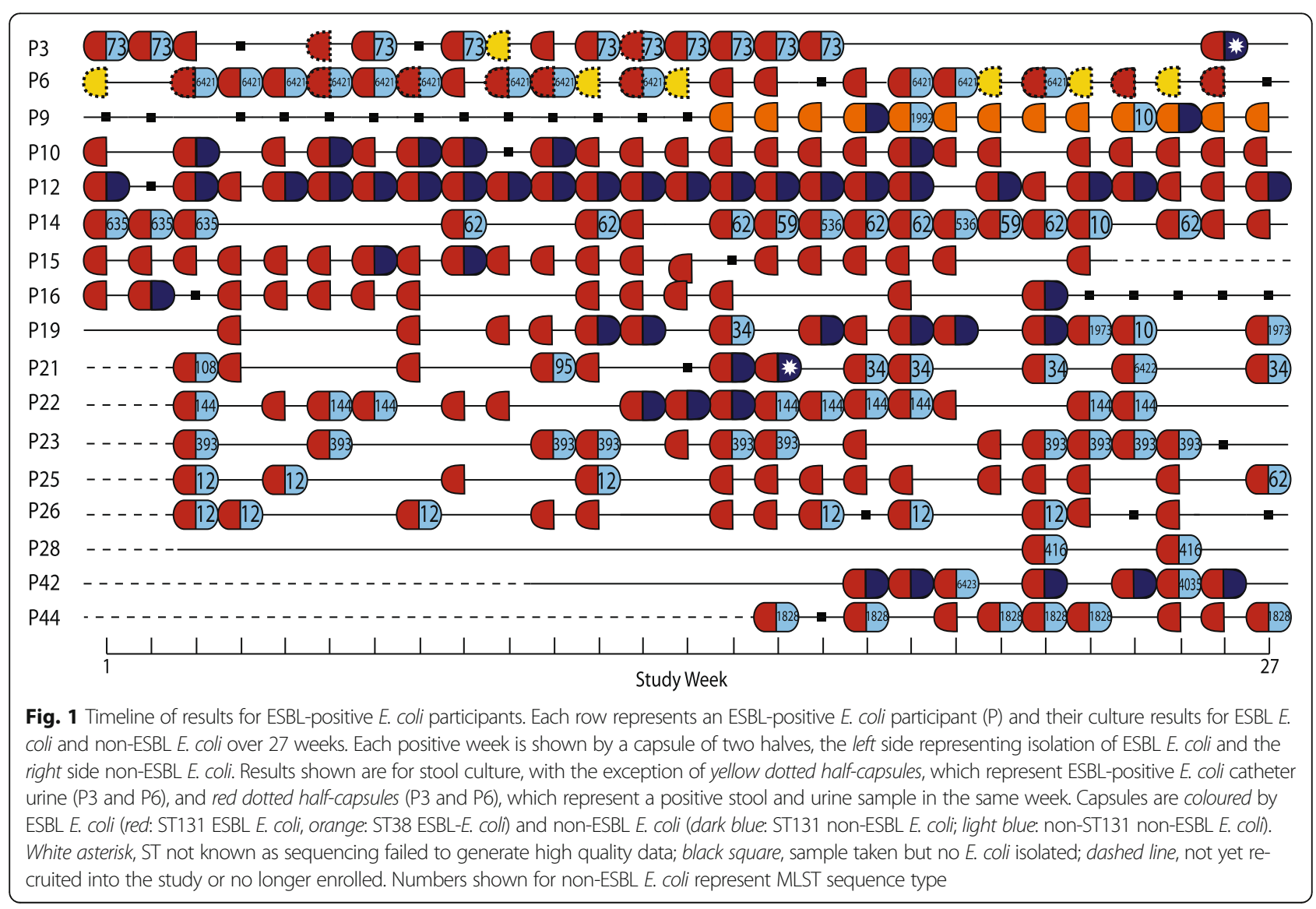


from stool (odds ratio $[\mathrm{OR}]=5.6,95 \%$ confidence interval $[\mathrm{CI}]=1.1-29.4, p=0.04-$ logistic regression model).

All 241 stools positive for ESBL E. coli were also cultured for non-ESBL E. coli). At least one isolate was cultured from all 17 ESBL-positive E. coli participants (isolated from 139/243 stools).

\section{Characterising E. coli isolates by MLST and ESBL encoding genes}

We sequenced 401 E. coli isolates (241 ESBL-E. coli from stool, 21 ESBL-E. coli from urine and 139 non-ESBL $E$. coli from stool). Two non-ESBL $E$. coli genomes were excluded from further analysis based on inadequate quality of sequence data. STs were identified from sequence data for the 399 remaining isolates. The 262 ESBL E. coli isolates were assigned to ST131 $(\mathrm{n}=249)$ or ST38 $(n=13)$. Sixteen participants carried ST131 ESBL E. coli and the remaining participant (P9) carried ST38 ESBL E. coli (Fig. 1). Non-ESBL E. coli were more genetically diverse, with 21 STs identified among the 137 isolates. The most common ST for non-ESBL E. coli was also ST131 ( $\mathrm{n}=48,35 \%)$, which was carried by $9 / 17$ participants, eight of whom were also positive for ST131
ESBL E. coli (Fig. 1). Seven participants were positive for more than one ST $($ median $=1 \mathrm{ST}$, range $=1-5 \mathrm{STs}$ ) and five STs were carried by more than one participant (ST10, ST12, ST34, ST62 and ST131) (Fig. 1). ESBL was encoded by bla $a_{\mathrm{CTX}-\mathrm{M}-15}$ in all 262 ESBL $E$. coli isolates.

\section{Genomic focus on E. coli ST131 from LTCF participants}

A maximum likelihood tree based on 797 single nucleotide polymorphisms (SNPs) in the core genome of 297 ST131 (see Additional file 1 for sequencing quality data) isolates from 17 participants compared with the reference E. coli NCTC13441 genome is shown in Fig. 2a. Isolates were distributed into multiple highly related clades, each corresponding to a positive participant. The pairwise SNP difference for isolates within each clade was in the range of 0-12 SNPs (median $=4$ SNPs). Based on the upper limit of 12 SNPs for within-host diversity, three groups of participants were defined as carrying the same clade: P19 and P42; P21, P28 and P42; and P19, P25 and P26, labelled as 1,2 and 3, respectively in Fig. 2a. These six participants all resided in unit 3. Additionally, three participants (P19, P12 and P42) each carried two distinct ST131 clades. In each case, these were

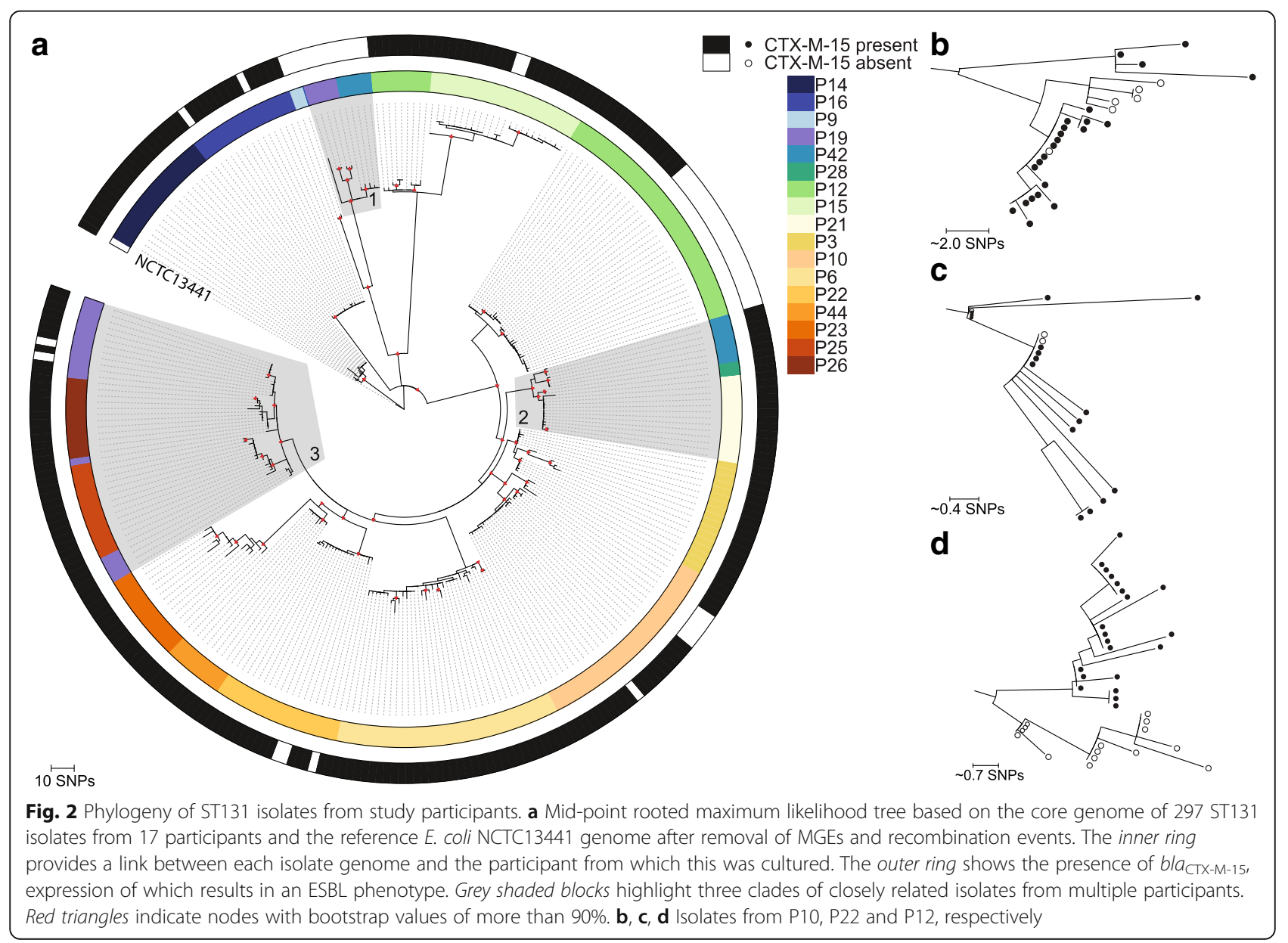


deemed to be independent based on their genetic distance (more than 100 SNPs apart in each case) and position in the phylogenetic tree.

Of the 16 participants who carried ESBL E. coli ST131, 11 carried only ST131 ESBL E. coli and eight carried a mixture of ST131 ESBL E. coli and ST131 non-ESBL E. coli. In addition, P9 carried non-ESBL E. coli ST131 in combination with ST38 ESBL E. coli. The degree of within-host relatedness between ESBL E. coli and nonESBL E. coli ST131 was illustrated by annotating the tree for the presence of $b l a_{\mathrm{CTX}-\mathrm{M}-15}$ (Fig. 2a), which revealed two patterns. Participants P10, P15, P16, P19 and P22 each carried clades that contained a mixture of highly related ESBL E. coli and non-ESBL E. coli (see Fig. 2b and $c$ for examples based on genomes from P10 and P22). By contrast, participant P19 carried genetically distinct ESBL E. coli and non-ESBL E. coli ST131 clades, which may be indicative of failure of $b l a_{\text {СTХ-M-15 }}$ to transfer in vivo from one clade to the other. P12 appeared to display carriage of both patterns and carried two distinct clades, one consisting of only isolates harbouring $b l a_{\mathrm{CTX}-\mathrm{M}-15}$ and a second clade containing both highly related ESBL and non-ESBL E. coli (Fig. 2d). Variation in the presence of beta-lactam ( $\left.b l a_{\text {OXA-1 }}\right)$, aminoglycoside (aac-(6')-Ib-cr), macrolide (mphA), trimethoprim ( $d f r A 17)$, tetracycline (tet $A)$, sulphonamide (sul1 and sul2) and streptomycin (strA and strB) resistance genes (Fig. 3) was also observed (Additional file 1). In five clades, loss/gain of other genes was associated with $b l a_{\mathrm{CTX}-\mathrm{M}-15}$, which is consistent with these residing on the same MGE. Analysis of the accessory (non-core) genome composition was performed to examine the context of the $b l a_{\mathrm{CTX}-\mathrm{M}-15}$ gene in ESBL $E$. coli isolates. In all $b l a_{\mathrm{CTX}-\mathrm{M}-15}$ positive isolates, the gene was associated with an MGE that resembled the peK499 plasmid [9], a hybrid of Incompatibility Type F replicons FIA and FII. The ESBL E. coli isolates revealed several variants of the peK499-like plasmid, as shown by the variable coverage after aligning the whole genome sequences against the peK499 plasmid (Fig. 3). Sequence coverage of the plasmid among the bla $a_{\mathrm{CTX}-\mathrm{M}-15}$ positive isolates was in the range of $54-83 \%$ (median $=76 \%$ ). Interestingly, in non-ESBL E. coli isolates, carriage of peK499-like plasmid sequences that lacked a region containing the

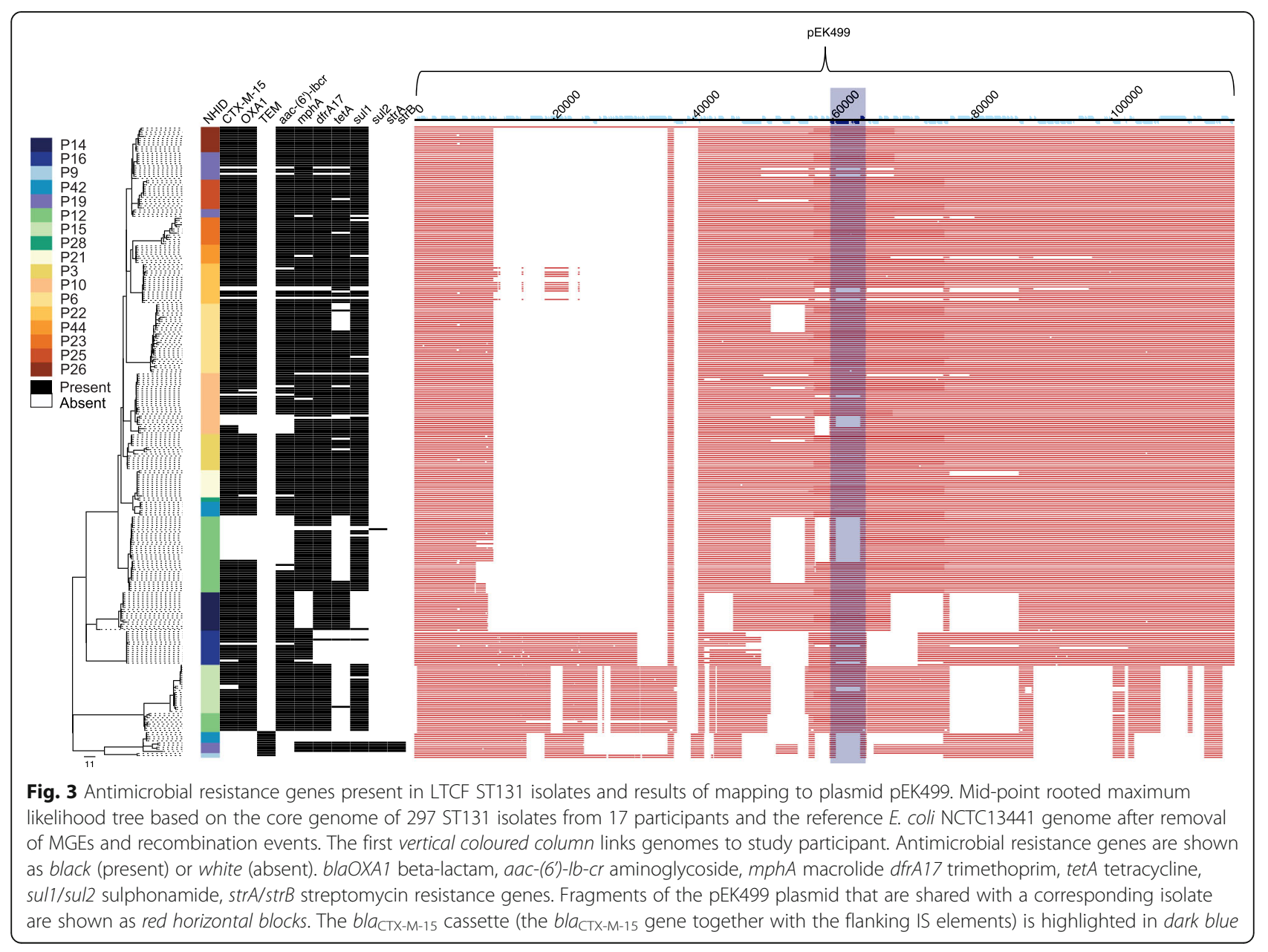


bla $a_{\mathrm{CTX}-\mathrm{M}-15}$ gene was also observed. In the peK499 plasmid, $b l a_{\text {CTX-M-15 }}$ is flanked on both sides by insertion sequence (IS26) transposase genes, suggesting a highly mobile cassette that may explain the loss and gain of ESBL status in the highly related ST131 isolates.

\section{Relatedness between ST131 from the LTCF and other healthcare settings}

Thirteen of the 17 ESBL-positive E. coli participants were admitted to the LTCF from the Cambridge University Hospitals NHS Foundation Trust (CUH) in the year prior to enrolment or during the study period. To determine the genetic relatedness between the study ST131 isolates and ST131 from patients at this hospital and further afield in England, we combined study genomes with whole-genome sequence data for 75 ST131 isolates (22 ESBL E. coli, 53 non-ESBL E. coli) from CUH and 146 ST131 isolates (52 ESBL E. coli, 94 non-ESBL E. coli) from ten hospitals across England, all associated with bloodstream infection. A subset of 30 study participant isolates were included in the analysis, consisting of one each of ESBL $E$. coli and non-ESBL $E$. coli from each lineage carried by participants. Data for these 251 ST131 isolates were used to construct a maximum likelihood tree based on 7676 SNPs in the core genome (Fig. 4). In this broader genetic context, isolates from LTCF participants fell into six clusters that were interspersed throughout the tree, although the majority $(20 / 30,16$ ESBL E. coli and four non-ESBL E. coli) resided in a single cluster containing isolates from 12 participants. The remaining clusters contained 1-4 isolates, from up to two different participants (Fig. 4). Three of these six LTCF clusters contained closely associated CUH isolates. For each of these three clusters we calculated the pairwise SNP differences between the LTCF and CUH isolates in the same cluster, which were in the ranges of $7-$ $66,19-67$ and 10-11 SNPs, respectively.

The 251 ST131 isolates were further categorised by defining the $\mathrm{fimH}$ allele, which demonstrated that 194 carried the fimH3O allele, placing them in clade $\mathrm{C}$ [57]. The remaining 57 isolates carried the following fimH alleles: H1 ( $n=3), H 12(n=2), H 17(n=30), H 25(n=2)$, H6 $(n=19)$ and unknown $(n=1)$. Clade $C$ has been further divided into three sub-clades termed $\mathrm{C} 0, \mathrm{C} 1$ and $\mathrm{C} 2$ based on point mutations [58-60]. The sub-clades of clade $\mathrm{C}$ were determined in this collection and are shown in Fig. 4, together with information on the mutations identified in Additional file 1.

\section{Discussion}

Prolonged or repeated antimicrobial use is a driver for the emergence of antimicrobial resistance and is an established risk factor for ESBL carriage [61-63]. We found that study participants who carried ESBL E. coli had been prescribed significantly more antimicrobials than those who did not. Residence in a LTCF is also a known risk factor for faecal carriage of ESBL E. coli [61]. In our six-month study, $38 \%$ of participants carried ESBL E. coli, which is consistent with carriage rates identified in previous studies from the UK $[28,29]$ and internationally [31, 64-66].

Serial sampling allowed us to describe within-host diversity of the same and different clades and lineages. WGS of healthcare-associated pathogens has begun to delineate the potential for complex within-host diversity [67-69]. To date, a small number of studies have investigated within-host diversity of ESBL $E$. coli using WGS [70, 71]. Sequencing of 16 bacterial colonies isolated from single stool samples obtained from eight children presenting to a hospital in Cambodia identified withinhost carriage of multiple $E$. coli lineages and variation in virulence and antibiotic resistance genes [70], supporting the findings in our study. Bayesian estimation of substitution rates in ST131 gave a value of $\sim 1$ SNP per genome per year [72]. The median level of within-host diversity in LTCF residents was four SNPs, which is consistent with long-term carriage and within-host evolution. We also analysed the relationship between ESBL $E$. coli and non-ESBL E. coli within individuals. Some participants carried ST131 ESBL E. coli and non-ESBL $E$. coli that were indistinguishable at the core genome level. This is consistent with previous studies that described loss and acquisition of either $b l a_{\mathrm{CTX}-\mathrm{M}-15}$ or the ESBL plasmid within an ST131 population and the presence of insertion and transposon sequences flanking the upstream and downstream regions of bla $a_{\text {CTX-M-15 }}[72,73]$.

The dominant ESBL E. coli lineage identified in our study was ST131, a finding consistent with previous studies [74-76]. We also isolated ST38 ESBL E. coli from one participant, which was acquired from an unknown reservoir during the study period. ST38 is commonly identified among human carriage and invasive isolates [76-78] and is increasingly being associated with bla $a_{\mathrm{OXA}-48}$, a beta-lactamase gene with significant carbapenamase activity, although this was not identified here $[79,80]$.

An important observation in this study was the value of contextual genetic databases in defining the relationship of ST131 isolates between study participants. Analysis of ST131 LTCF isolates alone suggested that participants carried several related but distinct populations, with three groups of participants carrying distinct clones of the same lineage. However, placing ST131 LTCF isolates into the genetic context of local and national ST131 collections revealed that the majority of isolates from LTCF participants clustered together, indicating acquisition of ST131 ESBL E. coli from a local lineage or a shared reservoir predating the study. The 


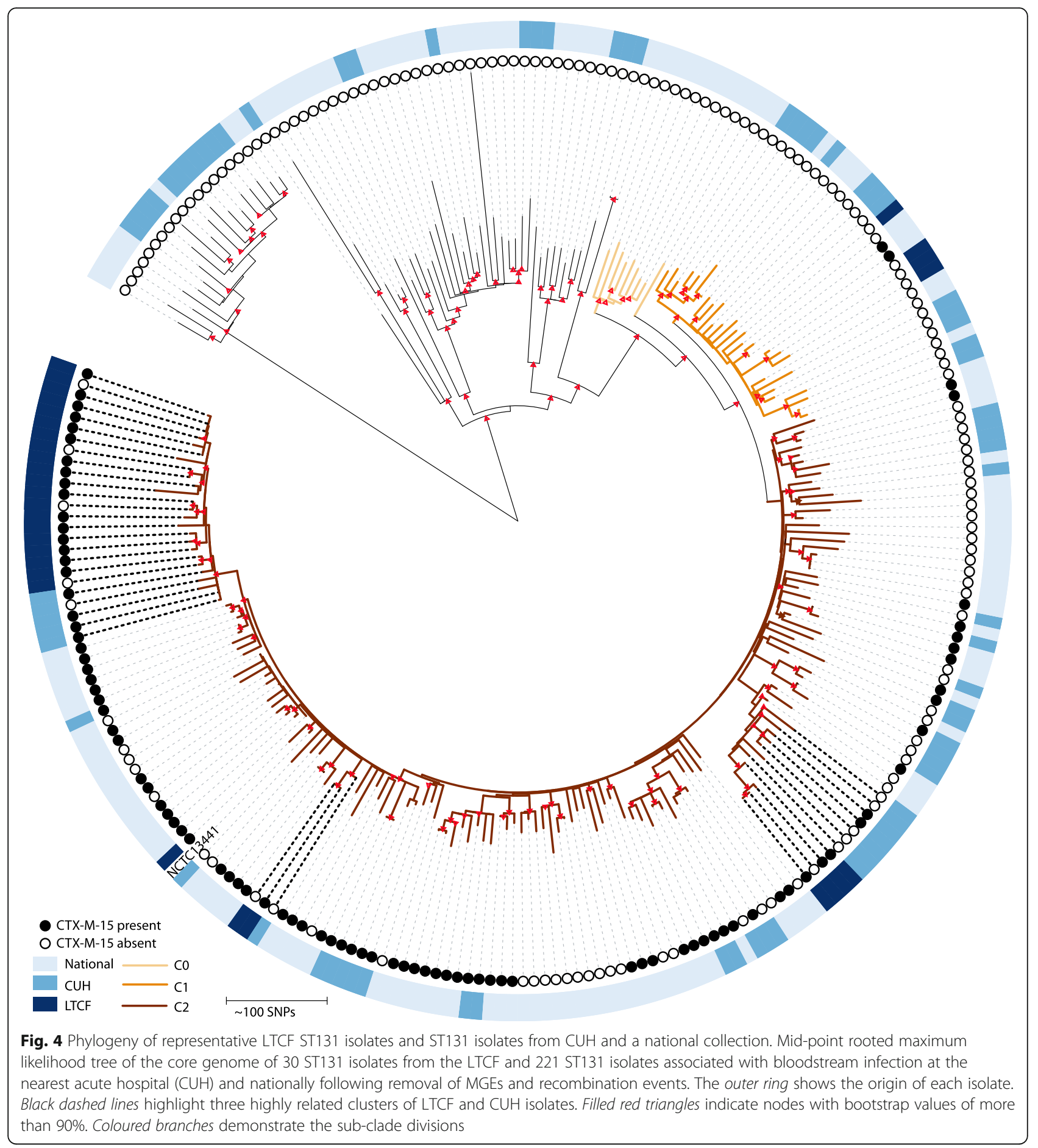

remaining LTCF isolates formed genetically distinct clades, indicating multiple introductions to the LTCF.

Our study had a number of limitations. A comprehensive understanding of carriage and transmission patterns requires $100 \%$ data capture, but we were only able to recruit $50 \%$ of the LTCF residents. Furthermore, our study design did not include sampling of healthcare workers, family members or the environment, all of which are potential donors or recipients of ESBL E. coli.

\section{Conclusions}

We confirmed that residents of a LTCF were a reservoir for multidrug-resistant E. coli and that ST131 dominated in this setting. We found evidence for a shared reservoir for ST131 within the LTCF, and between the LTCF and a 
nearby acute hospital. This suggests putative transmission within this broader healthcare network and underlines the importance of the interconnectivity in the spread of multidrug-resistant pathogens.

\section{Additional file}

Additional file 1: Additional information for the dataset used in this study. The additional file includes demographic information including location of isolation and sample type along with genome sequence QC data, results of genomic typing and screening for antimicrobial resistance genes. (XLSX $145 \mathrm{~kb}$ )

\section{Abbreviations}

BSAC: British Society for Antimicrobial Chemotherapy; CUH: Cambridge University Hospitals NHS Foundation Trust; ECDC: European Centre for Disease Prevention and Control; ESBL: Extended-spectrum beta-lactamase IQR: Interquartile range; LTCF: Long-term care facility; MALDI-TOF: Matrixassisted laser desorption/ionization time-of-flight mass spectrometry: MGE: Mobile genetic element; MLST: Multi-locus sequence type; PCR: Polymerase chain reaction; SNP: Single nucleotide polymorphism; ST: Sequence type; UK: United Kingdom; UPEC: Uropathogenic E. coli; WHO: World Health Organization

\section{Acknowledgements}

We gratefully acknowledge the contribution of the staff at the LTCF in sample collection and thank the patients who participated in the study. We thank Kirsty Ambridge and Angela Kidney for their technical assistance; and the library construction, sequencing and core informatics teams at the Wellcome Trust Sanger Institute. We thank the British Society for Antimicrobial Chemotherapy for providing isolates from the BSAC Resistance Surveillance Project. We thank Dr Direk Limmathurotsakul for his assistance with the statistical analysis. The views expressed in this publication are those of the author(s) and not necessarily those of the Department of Health or Wellcome Trust. MET is a Clinician Scientist Fellow supported by the Academy of Medical Sciences, The Health Foundation and the NIHR Cambridge Biomedical Research Centre.

\section{Funding}

This publication presents independent research supported by the Health Innovation Challenge Fund (WT098600, HICF-T5-342), a parallel funding partnership between the Department of Health and Wellcome Trust. The views expressed in this publication are those of the author(s) and not necessarily those of the Department of Health or Wellcome Trust.

\section{Availability of data and materials}

The datasets supporting the conclusions of this article are available in the European Nucleotide Archive under Project PRJEB7657: http://www.ebi.ac.uk/ ena/data/view/PRJEB7657, PRJEB4681: http://www.ebi.ac.uk/ena/data/view/ PRJEB4681 and Additional file 1 .

\section{Authors' contributions}

HJB, MET and SJP were involved in the design of the study. BB undertook bacterial identification and susceptibility testing. HJB extracted genomic DNA. HJB and KER were responsible for bioinformatics analysis of wholegenome sequence data. TK assisted in bioinformatics analysis and use of the CUH and BSAC collections. DJ assisted in bioinformatics analysis. HJB, KER and SJP wrote the manuscript. JP, MET and SJP were responsible for supervision and management of the study. All authors read and approved the final manuscript.

\section{Ethics approval and consent to participate}

The study protocol was approved by both the National Research Ethics Service (REC ref: 13/LO/1278) and the Cambridge University Hospitals NHS Foundation Trust Research and Development department (ref: A093007). Both ethics committees reviewed and approved the study protocol, consent forms and consultee information forms which expressly demonstrated that we would be including individuals with and without capacity in the study.
Participants with capacity provided informed consent prior to enrolment. Participants lacking capacity were enrolled following discussion with a relative or individual involved in their care (but not for remuneration). The study was carried out in a manner satisfying section 31 of the Mental Capacity Act, 2005, which enabled inclusion of residents lacking capacity in the study. The research study did not involve any intervention therefore the risk to residents was negligible. In addition, the research did not significantly interfere with resident's freedom of action or privacy. It was also not unduly invasive or restrictive. Residents lacking capacity were included in the study as the research could not be carried out as effectively without their inclusion, particularly in seeking evidence for transmission.

\section{Consent for publication}

All data have been anonymised therefore individual consent for publication is not applicable.

\section{Competing interests}

The authors declare that they have no competing interests.

\section{Publisher's Note}

Springer Nature remains neutral with regard to jurisdictional claims in published maps and institutional affiliations.

\section{Author details}

${ }^{1}$ Department of Medicine, University of Cambridge, Box 157, Addenbrooke's Hospital, Hills Road, Cambridge CB2 0QQ, UK. ${ }^{2}$ Wellcome Trust Sanger Institute, Wellcome Genome Campus, Hinxton, Cambridge CB10 1SA, UK. ${ }^{3}$ Cambridge Public Health England Microbiology and Public Health Laboratory, Box 236, Addenbrooke's Hospital, Hills Road, Cambridge CB2 OQQ, UK. ${ }^{4}$ British Society for Antimicrobial Chemotherapy, 53 Regent Place, Birmingham B1 3NJ, UK. ${ }^{5}$ Department of Medical Microbiology, Pathology Sciences Building 1, Southmead Hospital, Bristol BS10 5NB, UK. ${ }^{6}$ Cambridge University Hospitals NHS Foundation Trust, Hills Road, Cambridge CB2 OQQ, UK. ' London School of Hygiene and Tropical Medicine, London WC1E 7HT, UK

Received: 9 March 2017 Accepted: 4 July 2017

Published online: 25 July 2017

\section{References}

1. Davies SC. Annual Report of the Chief Medical Officer, Volume Two, 2011, Infections and the rise of antimicrobial resistance. London: Department of Health; 2013.

2. Public Health England. English surveillance programme for antimicrobial utilisation and resistance (ESPAUR) 2010 to 2014. London: Public Health England; 2015

3. Public Health England. Annual Epidemiological Commentary: Mandatory MRSA, MSSA and E. coli bacteraemia and C. difficile infection data 2013/14 London: Public Health England; 2014.

4. Coque T, Novais A, Carattoli A, Poirel L, Pitout J, Peixe L, et al. Dissemination of clonally related Escherichia coli strains expressing extended-spectrum beta-lactamase CTX-M-15. Emerg Infect Dis. 2008;14(2):195-200.

5. Nicolas-Chanoine MH, Blanco J, Leflon-Guibout V, Demarty R, Alonso MP, Canica MM, et al. Intercontinental emergence of Escherichia coli clone O25: H4-ST131 producing CTX-M-15. J Antimicrob Chemother. 2008;61(2):273-81.

6. Peirano G, Pitout JD. Molecular epidemiology of Escherichia coli producing CTX-M beta-lactamases: the worldwide emergence of clone ST131 O25:H4. Int J Antimicrob Agents. 2010:35(4):316-21.

7. Banerjee R, Robicsek A, Kuskowski MA, Porter S, Johnston BD, Sokurenko E, et al. Molecular epidemiology of Escherichia coli sequence type 131 and Its $\mathrm{H} 30$ and $\mathrm{H} 30-\mathrm{Rx}$ subclones among extended-spectrum-beta-lactamasepositive and -negative E. coli clinical isolates from the Chicago Region, 2007 to 2010. Antimicrob Agents Chemother. 2013;57(12):6385-8.

8. Peirano G, van der Bij AK, Gregson DB, Pitout JD. Molecular epidemiology over an 11-year period (2000 to 2010) of extended-spectrum betalactamase-producing Escherichia coli causing bacteremia in a centralized Canadian region. J Clin Microbiol. 2012:50(2):294-9.

9. Woodford N, Carattoli A, Karisik E, Underwood A, Ellington MJ, Livermore DM. Complete nucleotide sequences of plasmids pEK204, pEK499, and pEK516, encoding CTX-M enzymes in three major Escherichia coli lineages 
from the United Kingdom, all belonging to the international O25:H4-ST131 clone. Antimicrob Agents Chemother. 2009;53(10):4472-82.

10. Lautenbach E, Patel JB, Bilker WB, Edelstein PH, Fishman NO. Extendedspectrum Beta-lactamase-producing Escherichia coli and Klebsiella pneumoniae: Risk factors for infection and impact of resistance on outcomes. Clin Infect Dis. 2001;32(8):1162-71.

11. Melzer M, Petersen I. Mortality following bacteraemic infection caused by extended spectrum beta-lactamase (ESBL) producing E. coli compared to non-ESBL producing E. coli. J Infect. 2007;55(3):254-9.

12. Schwaber MJ, Carmeli Y. Mortality and delay in effective therapy associated with extended-spectrum beta-lactamase production in Enterobacteriaceae bacteraemia: a systematic review and meta-analysis. J Antimicrob Chemother. 2007;60(5):913-20

13. Woodford N, Ward ME, Kaufmann ME, Turton J, Fagan EJ, James D, et al. Community and hospital spread of Esherichia coli producing CTX-M extended-spectrum beta-lactamases in the UK. J Antimicrob Chemother. 2004:54(4):735-43.

14. Saladin M, Cao VTB, Lambert T, Donay JL, Herrmann JL, Ould-Hocine Z, et al. Diversity of CTX-M beta-lactamases and their promoter regions from Enterobacteriaceae isolated in three Parisian hospitals. FEMS Microbiol Lett. 2002;209(2):161-8.

15. Hernandez JR, Martinez-Martinez L, Canton R, Coque TM, Pascual A. Spanish Group for Nosocomial Infections. Nationwide study of Escherichia coli and Klebsiella pneumoniae producing extended-spectrum beta-lactamases in Spain. Antimicrob Agents Chemother. 2005;49(5):2122-5.

16. Livermore DM, Canton R, Gniadkowski M, Nordmann P, Rossolini GM, Arlet G, et al. CTX-M: changing the face of ESBLs in Europe. J Antimicrob Chemother. 2007;59(2):165-74.

17. Munday CJ, Xiong J, Li C, Shen D, Hawkey PM. Dissemination of CTX-M type beta-lactamases in Enterobacteriaceae isolates in the People's Republic of China. Int J Antimicrob Agents. 2004;23(2):175-80.

18. Lu PL, Liu YC, Toh HS, Lee YL, Liu YM, Ho CM, et al. Epidemiology and antimicrobial susceptibility profiles of Gram-negative bacteria causing urinary tract infections in the Asia-Pacific region: 2009-2010 results from the Study for Monitoring Antimicrobial Resistance Trends (SMART). Int J Antimicrob Agents. 2012:40(S1):S37-43.

19. Kazmierczak KM, Lob SH, Hoban DJ, Hackel MA, Badal RE, Bouchillon SK. Characterization of extended-spectrum beta-lactamases and antimicrobial resistance of Klebsiella pneumoniae in intra-abdominal infection isolates in Latin America, 2008-2012. Results of the Study for Monitoring Antimicrobial Resistance Trends. Diagn Microbiol Infect Dis. 2015;82(3):209-14.

20. Day MJ, Doumith M, Abernethy J, Hope R, Reynolds R, Wain J, et al. Population structure of Escherichia coli causing bacteraemia in the UK and Ireland between 2001 and 2010. J Antimicrob Chemother. 2016;71(8):2139-42

21. European Centre for Disease Prevention. Antimicrobial resistance surveillance in Europe 2013. Annual Report of the European Antimicrobial Resistance Surveillance Network (EARS-Net). Stockholm: ECDC; 2014

22. O'Neill J. The review on antimicrobial resistance. London: Department of Health; 2016.

23. World Health Organization. Antimicrobial Resistance Global Report on Surveillance. Geneva: WHO; 2014.

24. World Health Organization. Global Antimicrobial Resistance Surveillance System: Manual for early implementation. Geneva: WHO; 2015.

25. World Health Organization. Central Asian and Eastern European Surveillance of Antimicrobial Resistance. Geneva: WHO; 2015.

26. Donaldson L. Getting ahead of the curve: A strategy for combatting infectious diseases. A report by the Chief Medical Officer. London: Department of Health; 2002.

27. Randle J, Bellamy E. Infection control nurses' perceptions of managing MRSA in the community. J Infect Prev. 2011;12:142-5.

28. Ludden C, Cormican M, Vellinga A, Johnson JR, Austin B, Morris D. Colonisation with ESBL-producing and carbapenemase-producing Enterobacteriaceae, vancomycin-resistant enterococci, and meticillinresistant Staphylococcus aureus in a long-term care facility over one year. BMC Infect Dis. 2015;15(1):168.

29. Rooney PJ, O'Leary MC, Loughrey AC, McCalmont M, Smyth B, Donaghy P, et al. Nursing homes as a reservoir of extended-spectrum beta-lactamase (ESBL)-producing ciprofloxacin-resistant Escherichia coli. J Antimicrob Chemother. 2009;64(3):635-41.

30. Karanika S, Karantanos T, Arvanitis M, Grigoras C, Mylonakis E. Fecal colonization with extended-spectrum beta-lactamase-producing
Enterobacteriaceae and risk factors among healthy individuals: A systematic review and metaanalysis. Clin Infect Dis. 2016;63(3):310-8.

31. Gruber I, Heudorf U, Werner G, Pfeifer Y, Imirzalioglu C, Ackermann H, et al. Multidrug-resistant bacteria in geriatric clinics, nursing homes, and ambulant care-prevalence and risk factors. Int J Med Microbiol. 2013;303(8):405-9.

32. Luvsansharav UO, Hirai I, Niki M, Nakata A, Yoshinaga A, Yamamoto A, et al. Fecal carriage of CTX-M beta-lactamase-producing Enterobacteriaceae in nursing homes in the Kinki region of Japan. Infect Drug Resist. 2013;6:67-70.

33. Valenza G, Nickel S, Pfeifer Y, Pietsch M, Voigtlander E, Lehner-Reindl V, et al. Prevalence and genetic diversity of extended-spectrum $\beta$-lactamase (ESBL)producing Escherichia coli in nursing homes in Bavaria. Germany Vet Microbiol. 2017;200:138-41.

34. Brodrick HJ, Raven KE, Harrison EM, Blane B, Reuter S, Török ME, et al. Whole-genome sequencing reveals transmission of vancomycin-resistant Enterococcus faecium in a healthcare network. Genome Med. 2016:8(1):4

35. Zerbino DR, Birney E. Velvet: algorithms for de novo short read assembly using de Bruijn graphs. Genome Res. 2008;18(5):821-9.

36. Boetzer M, Henkel CV, Jansen HJ, Butler D, Pirovano W. Scaffolding preassembled contigs using SSPACE. Bioinformatics. 2011;27(4):578-9.

37. Boetzer M, Henkel CV, Jansen HJ, Butler D, Pirovano W. Toward almost closed genomes with GapFiller. Genome Biol. 2012;13(6):R56.

38. Seemann T. Prokka: rapid prokaryotic genome annotation. Bioinformatics. 2014;30(14):2068-9.

39. Pruitt KD, Tatusova T, Brown GR, Maglott DR. NCBI Reference Sequences (RefSeq): current status, new features and genome annotation policy. Nucleic Acids Res. 2012;40(Database issue):D130-5.

40. Wirth T, Falush D, Lan R, Colles F, Mensa P, Wieler LH, et al. Sex and virulence in Escherichia coli: an evolutionary perspective. Mol Microbiol. 2006;60(5):1136-51.

41. Page AJ, Tayloer B, Keane JA. Multilocus sequence typing by blast from de novo assemblies against PubMLST. The Journal of Open Source Software. 2016. https://github.com/sanger-pathogens/mlst_check. Accessed Nov 2015.

42. Jolley KA, Maiden MC. BIGSdb: Scalable analysis of bacterial genome variation at the population level. BMC Bioinformatics. 2010;11:595.

43. Ponstigl H. SMALT: Pairwise Sequence Alignment Program. 2010. Available at: http://ftp.sanger.ac.uk/pub/resources/software/smalt/smalt-manual-0.6.2. pdf. Accessed Nov 2015

44. Li H, Handsaker B, Wysoker A, Fennell T, Ruan J, Homer N, et al. The Sequence Alignment/Map format and SAMtools. Bioinformatics. 2009;25(16): 2078-9.

45. Zhou Y, Liang Y, Lynch KH, Dennis JJ, Wishart DS. PHAST: a fast phage search tool. Nucleic Acids Res. 2011;39(Web Server issue):W347-52.

46. Croucher NJ, Page AJ, Connor TR, Delaney AJ, Keane JA, Bentley SD, et al. Rapid phylogenetic analysis of large samples of recombinant bacterial whole genome sequences using Gubbins. Nucleic Acids Res. 2015;43(3), e15.

47. Stamatakis A. RAxML-VI-HPC: maximum likelihood-based phylogenetic analyses with thousands of taxa and mixed models. Bioinformatics. 2006; 22(21):2688-90.

48. Rambaut A. FigTree: Tree Figure Drawing Tool, version 1.4.2. 2014. Available at: http://tree.bio.ed.ac.uk/software/figtree/. Accessed Nov 2015.

49. Letunic I, Bork P. Interactive tree of life (iTOL) v3: an online tool for the display and annotation of phylogenetic and other trees. Nucleic Acids Res. 2016:44(W1):W242-5.

50. Zankari E, Hasman H, Consentino S, Vestergaard M, Rasmussen S, Lund O, et al. Identification of acquired antimicrobial resistance genes. J Antimicrob Chemother. 2012;67(11):2640-4.

51. Weissman SJ, Johnson TR, Tchesnokova V, Billig M, Dykhuizen D, Ridell K, et al. High-resolution two-locus clonal typing of extraintestinal pathogenic Escherichia coli. Appl Environ Microbiol. 2012;78(5):1353-60.

52. Colpan A, Johnston B, Porter S, Clabots C, Anway R, Thao L, et al. Escherichia coli sequence type 131 (ST131) subclone H30 as an emergent multidrug resistant pathogen among US veterans. Clin Infect Dis. 2013;57(9):1256-65.

53. Gouy M, Guindon S, Gascuel O. SeaView version 4: A multiplatform graphical user interface for sequence alignment and phylogenetic tree building. Mol Biol Evol. 2010;27(2):221-4.

54. Harris SR, Feil E, Holden MTG, Quail MA, Nicker EK, Chantratita N, et al. Evolution of MRSA during hospital transmission and intercontinental spread. Science. 2010;327(5964):469-74.

55. Kurtz S, Phillippy A, Delcher AL, Smoot M, Shumway M, Antonescu C, et al. Versatile and open software for comparing large genomes. Genome Biol. 2004;5:R12. 
56. Inouye M, Dashnow H, Raven L-A, Schultz MB, Pope BJ, Tomita T, et al. SRST2: Rapid genomic surveillance for public health and hospital microbiology labs. Genome Med. 2014;6:90.

57. Petty NK, Ben Zakour NL, Stanton-Cook M, Skippington E, Totsika M, Forde BM, et al. Global dissemination of a multidrug resistant Escherichia coli clone. Proc Natl Acad Sci U S A. 2014;111(15):5694-9.

58. Ben Zakour NL, Alsheikh-Hussain AS, Ashcroft MM, Nguyen TKN, Roberts LW, Stanton-Cook M, et al. Sequential acquisition of virulence and fluoroquinolone resistance has shaped the evolution of Escherichia coli ST131. MBio. 2016;7(2):e00347-16.

59. Johnson JR, Tchesnokova V, Johnston B, Clabots C, Roberst PL, Billig M, et al. Abrupt emergence of a single dominant multidrug-resistant strain of Escherichia coli. J Infect Dis. 2013;207:919-28.

60. Price L, Johnson J, Aziz M, Clabots C, Johnston B, Tchesnokova V, et al. The epidemic of extended-spectrum-beta-lactamase-producing Escherichia coli ST131 is driven by a single highly pathogenic subclone, H30-Rx. MBio. 2013; 4(6):e00377-13.

61. Ben-Ami R, Rodrigues-Baño J, Arslan H, Pitout JD, Quentin C, Calbo ES, et al. A multinational survey of risk factors for infection with extended-spectrum beta-lactamase-producing enterobacteriaceae in nonhospitalized patients. Clin Infect Dis. 2009;49(5):682-90.

62. Colodner R, Rock W, Chazan B, Keller N, Guy N, Sakran W, et al. Risk factors for the development of extended-spectrum beta-lactamase-producing bacteria in nonhospitalized patients. Eur J Clin Microbiol Infect Dis. 2004 23(3):163-7.

63. Jans B, Schoevaerdts D, Huang TD, Berhin C, Latour K, Bogaerts P, et al. Epidemiology of multidrug-resistant microorganisms among nursing home residents in Belgium. PLoS One. 2013;8(5), e64908.

64. Jallad MA, Naoufal R, Irani J, Azar E. Extended spectrum beta-lactamase carriage state among elderly nursing home residents in Beirut. ScientificWorld Journal. 2015;2015:987580.

65. Pop-Vicas A, Mitchel SL, Kandel R, Schreiber R, D'Agata EM. Multidrugresistant gram-negative bacteria in a long-term care facility: prevalence and risk factors. J Am Geriatr Soc. 2008;56(7):1276-80.

66. Zhao SY, Zhang J, Zhang YL, Wang YC, Xiao SZ, Gu FF, et al. Epidemiology and risk factors for faecal extended-spectrum beta-lactamase-producing Enterobacteriaceae (ESBL-E) carriage derived from residents of seven nursing homes in western Shanghai, China. Epidemiol Infect. 2016;144(4):695-702.

67. Harris SR, Cartwright EJP, Török ME, Holden MTG, Brown NM, Ogilvy-Stuart AL. Whole-genome sequencing for analysis of an outbreak of meticillinresistant Staphylococcus aureus: a descriptive study. Lancet Infect Dis. 2013; 13(2):130-6.

68. Paterson GK, Harrison EM, Murray GG, Welch JJ, Warland JH, Holden MT, et al. Capturing the cloud of diversity reveals complexity and heterogeneity of MRSA carriage, infection and transmission. Nat Commun. 2015;6:6560.

69. Young BC, Golubchik T, Batty EM, Fung R, Larner-Svensson H, Votintseva AA, et al. Evolutionary dynamics of Staphylococcus aureus during progression from carriage to disease. PNAS. 2012;109(12):4550-5.

70. Salipante SJ, Roach DJ, Kitzman JO, Snyder MW, Stackhouse B, Butler-Wu SM, et al. Large-scale genomic sequencing of extraintestinal pathogenic Escherichia coli strains. Genome Res. 2015;25(1):119-28.

71. Stoesser N, Sheppard AE, Moore CE, Golubchik T, Parry CM, Nget P, et al. Extensive within-host diversity in fecally carried extended-spectrum betalactamase-producing Escherichia coli: implications for transmission analyses. J Clin Microbiol. 2015;53(7):2122-31.

72. Stoesser N, Sheppard AE, Pankhurst L, De Maio N, Moore CE, Sebra R, et al. Evolutionary history of the global emergence of the Escherichia coli epidemic clone ST131. MBio. 2016;7(2), e02162.

73. Doumith M, Dhanji H, Ellginton MJ, Hawkey P, Woodford N. Characterization of plasmids encoding extended-spectrum beta-lactamases and their addiction systems circulating among Escherichia coli clinical isolates in the UK. J Antimicrob Chemother. 2012;67(4):878-85.

74. Banerjee R, Johnston B, Lohse C, Porter SB, Clabots C, Johnson JR. Escherichia coli sequence type 131 is a dominant, antimicrobial-resistant clonal group associated with healthcare and elderly hosts. Infect Control Hosp Epidemiol. 2013;34(4):361-9.

75. Lau SH, Kaufmann ME, Livermore DM, Woodford N, Willshaw GA, Cheasty T, et al. UK epidemic Escherichia coli strains A-E, with CTX-M-15 betalactamase, all belong to the international O25:H4-ST131 clone. J Antimicrob Chemother. 2008:62(6):1241-4.
76. Peirano G, van der Bij AK, Freeman JL, Poirel L, Nordmann P, Costello M et al. Characteristics of Escherichia coli sequence type 131 isolates that produce extended-spectrum beta-lactamases: global distribution of the H30-Rx sublineage. Antimicrob Agents Chemother. 2014;58(7):3762-7.

77. Alghoribi MF, Gibreel TM, Farnham G, Al Johani SM, Balkhy HH, Upton M. Antibiotic-resistant ST38, ST131 and ST405 strains are the leading uropathogenic Escherichia coli clones in Riyadh, Saudi Arabia. J Antimicrob Chemother. 2015;70(10):2757-62.

78. Müller A, Stephan R, Nüesch-Inderbinen M. Distribution of virulence factors in ESBL-producing Escherichia coli isolated from the environment, livestock, food and humans. Sci Total Environ. 2016:541:667-72.

79. Poirel L, Bernabeu S, Fortineau N, Podglajen I, Lawrence C, Nordmann P. Emergence of OXA-48-producing Escherichia coli clone ST38 in France. Antimicrob Agents Chemother. 2011;55(10):4937-8.

80. Turton JF, Doumith M, Hopkins KL, Perry C, Meunier D, Woodford N. Clonal expansion of Escherichia coli ST38 carrying chromosomally-integrated OXA48 carbapenemase gene. J Med Microbiol. 2016;65(6):538-46.

\section{Submit your next manuscript to BioMed Central and we will help you at every step:}

- We accept pre-submission inquiries

- Our selector tool helps you to find the most relevant journal

- We provide round the clock customer support

- Convenient online submission

- Thorough peer review

- Inclusion in PubMed and all major indexing services

- Maximum visibility for your research

Submit your manuscript at www.biomedcentral.com/submit
) Biomed Central 